\title{
CONTROVERSIES
}

Ramon M. Esclamado, MD, Section Editor

\section{MANAGEMENT OF TONSIL CANCER}

\author{
Consultants: Carol R. Bradford, MD, Neal Futran, MD, DMD, \\ Glenn Peters, MD
}

A 43-year old man is examined for evaluation of a persistent, right-sided sore throat for 4 months, and a painless, slowly enlarging right neck mass for 3 months. On further questioning, he has right otalgia, but denies dysphagia, odynophagia, hemoptysis, hoarseness, or weight loss. He has a 40 pack/year smoking history, but is otherwise in good health.

Physical examination reveals a $3 \mathrm{~cm} \times 3 \mathrm{~cm}$ exophytic mass involving the right tonsillar fossa and posterior tonsillar pillar. There is no trismus, the tongue base and soft palate are not involved, and his teeth are in good repair. The remainder of the head and neck examination is remarkable only for a firm, freely mobile $4 \mathrm{~cm} \times 4 \mathrm{~cm}$ right level II lymph node.

A chest radiograph is normal, and a neck $\mathrm{CT}$ is shown in Figure 1. Examination under anesthesia confirmed the above findings, and no synchro-

Readers are invited to submit particularly difficult cases for consideration to Ramon M. Esclamado, MD, Editor, Controversies, Department of Otolaryngology and Communicative Disorders/A71, The Cleveland Clinic Foundation, 9500 Euclid Avenue, Cleveland, OH 44195.

From the Department of Otolaryngology, University of Michigan Medical Center, Ann Arbor, Michigan (Dr. Bradford); the Department of Otolaryngology, University of Washington, Seattle (Dr. Futran); Division of Otolaryngology, University of Alabama, Birmingham (Dr. Peters)

Accepted for publication April 27, 1999

Head \& Neck 21: 657-662, 1999

CCC 1043-3074/99/0070657-06

(C) 1999 John Wiley \& Sons, Inc nous primaries are seen in the upper aerodigestive tract and esophagus. The biopsy is positive for invasive moderately well-differentiated squamous cell cancer, therefore staged as T2 N2a M0.

1. What treatment options are available for this patient that can be offered outside of a clinical trial?

Dr. Bradford: This 43-year old male with a T2 N2a M0 moderately differentiated squamous cell carcinoma of the right tonsillar fossa could be treated either with primary surgery plus postoperative radiation therapy or radiation therapy primarily followed by a modified neck dissection. A third alternative would be radiation therapy alone and close monitoring of the primary site and neck.

Dr. Futran: Treatment options include (1) external beam radiation therapy alone to the primary lesion and both sides of the neck followed by right modified neck dissection, (2) external beam radiation therapy as above with brachytherapy to the primary site followed by right modified radical neck dissection, (3) surgical extirpation of the primary lesion with a modified radical neck dissection and likely flap reconstruction followed by external beam radiation therapy, or (4) for T2 lesions, I do not recommend chemotherapy con- 


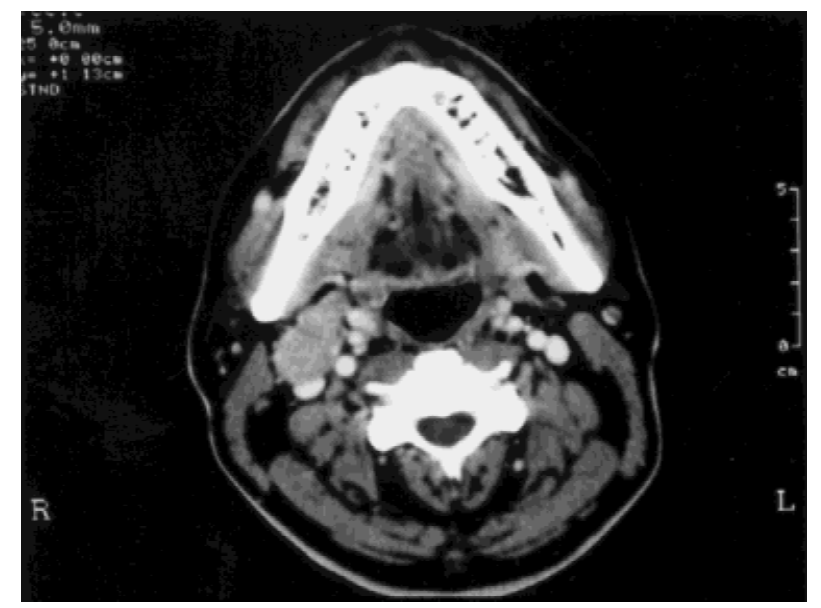

FIGURE 1. Axial CT scan with contrast demonstrating level II node with superficial primary tumor and no pterygoid muscle of mandible involvement.

comitant with radiotherapy or as an adjunct to radiation therapy.

Dr. Peters: Outside of a protocol setting, the treatment options include radiation therapy or surgery. I would be reluctant to use chemotherapy as an additional treatment modality unless the patient was on a protocol of some sort. Radiation could be given as a single daily fraction, but I feel that this patient would likely receive an accelerated fractionation treatment program (BID, concomitant boost, etc.). Surgery would include resection of the primary along with neck dissection and free flap reconstruction. The need for postoperative radiation would be assessed based on the path report.

\section{Are there any tumor characteristics that are helpful in determining a preferred treatment option?}

Dr. Futran: (1) Location of the tumor in the tonsillar fossa: tumors located within the tonsillar fossa tend to have a higher rate of metastasis than tumors arising from the anterior pillar, but also tend to be more radiosensitive. Tumors arising from the anterior tonsillar pillar tend to be more well differentiated and may extend to the retromolar trigone, making surgery a more attractive primary option. Further, if the tumor extends onto the tongue base, primary radiation therapy has a poorer control rate and would influence me to use surgical extirpation as the primary option. (2) Several studies suggest that exophytic tonsil tumors are much more radiosensitive than ulcerated or indurated tumors. (3) As most tonsillar fossa tumors are moderate- to welldifferentiated lesions, the degree of differentiation would not significantly influence my decision making. (4) The presence of clinical trismus associated with the tumors suggests pterygoid muscle involvement and would influence me toward a primary surgical treatment plan.

Dr. Peters: Although there is considerable effort being put forth trying to identify pretreatment prognostic indicators, at this point in time I still rely chiefly on the clinical stage in making treatment recommendations. Despite the obvious weaknesses and shortcomings that exist with the current staging system, there has not yet been identified a reliable biologic or molecular marker to assist us in making these decisions.

Dr. Bradford: A lymphoepithelioma or poorly differentiated carcinoma of the tonsillar fossa should be more radiosensitive than moderately or well-differentiated tumors. A thin or exophytic tumor probably also would be more radiosensitive. These factors would include my decision to treat with primary radiotherapy, particularly the lymphoepithelioma histology.

\section{Assuming that the patient has no treat- ment biases, what would be your treatment recommendation?}

Dr. Peters: I think that this is an ideal patient for which I would recommend treatment on a protocol using combined modality chemotherapy and radiation therapy with surgery held in the salvage mode. If protocol treatment is not an option, then I would recommend accelerated fraction radiation therapy, again with surgery held for salvage. The patient would be followed very closely during therapy, monitoring him for an objective response at the end of two cycles of chemotherapy or 50 Gy of radiation. If an appropriately good response is noted, then I would feel fairly comfortable in continuing nonsurgical therapy to its completion. If a less than adequate response is seen in this patient then we would go on to surgery. At the conclusion of therapy, any remaining mass in the neck, demonstrated either by physical examination or CT, would need to be removed with a neck dissection. I have been using selective neck dissections (leaving level $\mathrm{V}$ undissected) more in this setting as I have found a large number of patients who have pathologically negative necks. Another important aspect of treatment is 
nutrition and I would place a PEG prior to the initiation of any therapy.

My reason for choosing this treatment approach is that it provides the patient with the best chance for preserving nearly normal swallowing and speech. As long as the patient is monitored closely and his response to therapy is carefully established I do not feel that oncologic control is compromised.

Dr. Bradford: The advantage of surgery and postoperative radiation therapy is that both the primary site and neck receive combined modality treatment. Historically, tonsillar fossa lesions have been treated with radiation therapy primarily. However, several reports indicate that such an approach may decrease locoregional control. ${ }^{1,2}$ Given the improved results for reconstruction following surgical excision of tonsillar carcinomas, I favor an approach that involves primary surgery followed by postoperative radiation therapy. Another factor in this treatment preference is the opportunity for an in-continuity neck dissection. There is very little distance between the primary site and the level II lymph node on this CT scan. On several occasions we have identified high retropharyngeal nodes that were positive for squamous carcinoma on histopathologic analysis. Resection of these nodes followed by postoperative radiation therapy seems to provide the best chance for locoregional control of disease. Furthermore, histopathologic analysis of the lymph nodes provides an opportunity to enroll patients in randomized prospective protocols comparing radiation therapy to chemotherapy plus radiation therapy for high-risk pathology (numerous positive nodes, extracapsular extension, close surgical margins).

Dr. Futran: For this particular patient and tumor, external beam radiation therapy to a mean total dose of 6,500 centigray to the primary site and both necks, will provide an $80 \%$ chance of local control and an excellent functional result. The patient would also have a planned postradiation right modified radical neck dissection. Although primary surgical extirpation and neck dissection is an acceptable option followed by radiation therapy, the defect would likely require a flap reconstruction and possible resection of the angle of the mandible. Although the functional result with proper reconstruction would be quite good, if radiation therapy alone controls the pri- mary tumor, the ultimate functional result would be better.

4. If surgery followed by postoperative radiation was recommended, how would the primary tumor be approached, and what type of neck dissection would you perform?

Dr. Bradford: The specifics of this treatment approach are as follows. I have a very low threshold for mandibulotomy to improve visualization for surgical extirpation. In a teaching environment, the mandibulotomy combined with lip split provides excellent exposure with minimal morbidity. One can fashion the mandibulotomy to preserve mental nerve continuity. A stairstep mandibulotomy is planned medial to the mental foramen. Prior to bony cuts, a 3D, high profile, 2.0 $\mathrm{mm}$ plate is fashioned with three bicortical screws on either side of the planned mandibulotomy. The lip split incision is performed in the midline with a step at the vermilliocutaneous junction and a Z-plasty between this junction and the chin crease. I prefer the chin to be split in the midline rather than around the chin subunit. If the patient does not have tight dental junctions, one can perform the mandibulotomy between teeth (usually between the canine and lateral incisor). The lingual nerve can be preserved provided it is not involved with tumor, although this does limit the swing of the mandible. Radical resection of tumor can then be accomplished with 2 -cm margins and frozen section control.

The neck dissection I would recommend for a 4-cm jugulodigastric node is a modified versus a radical neck dissection. I would plan to sacrifice the sternocleidomastoid muscle. If cranial nerve $\mathrm{XI}$ is not involved with tumor it can be saved. Direct tumor involvement would dictate sacrifice of the XIth cranial nerve. The internal or external jugular vessels can be saved for microvascular anastomosis only if free from tumor. If not, contralateral vessel dissection (usually performed in the setting of a limited selective neck dissection) would be required if free tissue transfer is planned.

Optimum reconstruction for this anticipated 7 $\mathrm{cm} \times 7 \mathrm{~cm}$ defect of the tonsillar fossa and surrounding region could be achieved with either a platysma myocutaneous flap or a radial forearm free fasciocutaneous flap. The platysma myocutaneous flap, however, is not advisable in the setting of modified neck dissection when the sternocleidomastoid muscle is not preserved. ${ }^{3}$ The rationale for this caveat is lack of carotid artery 
protection due to coverage with only a thin cutaneous flap. Therefore, a radial forearm free fasciocutaneous flap would be ideal for reconstruction of this defect. Presumably the patient would lose approximately half of the soft palate. Functional reconstruction can be achieved by narrowing the nasopharyngeal port with primary closure of the posterior aspect of the tonsillar/soft palate defect (unpublished data, Chepeha DB and Teknos TN). The radial forearm free flap can then be sutured into the anterior aspect of the defect. Utilizing this technique has allowed functional restoration without velopharyngeal insufficiency in a series of patients with advanced tonsillar carcinoma.

Full course radiation therapy would be advised to commence 4 to 6 weeks postoperatively. Evidence of extracapsular extension on nodal pathology would make the patient eligible for randomized protocols comparing radiation alone with chemotherapy plus radiation.

Dr. Futran: First, I would assess whether the tumor is fixed to the mandible. If there is any adherence to the mandible at the angle region, to get a proper margin, I would perform a composite resection including the angle of the mandible via lip split approach. If the tumor is not adherent to the mandible, I would approach this patient by lip split and midline mandibular split approach, resect the primary tumor in continuity with a modified radical neck dissection, sparing only the spinal accessory nerve. The optimal reconstruction would be with a sensate radial forearm fasciocutaneous free flap if the mandible is spared. If the angle of the mandible is resected, I would use a radial forearm osteofasciocutaneous sensate free flap.

Dr. Peters: I would approach the primary tumor with a mandible sparing procedure such as a median mandibulotomy. The neck disease is fairly bulky and I feel it would be difficult to preserve his spinal accessory nerve and the internal jugular vein. Therefore, I feel a classic radical neck dissection would be the operation of choice.

5. If definitive radiation or chemoradiation were used first, and a complete locoregional response was observed, would you perform a planned neck dissection?

Dr. Futran: Our philosophy at the University of Washington is to treat the neck based on the primary nodal presentation, not specifically on the response to treatment. Several studies, particularly in the European literature, suggest that even with complete clinical response of the neck to radiation therapy and chemotherapy protocols, occult disease may still be present. Until there is evidence that posttherapy necks that are clinically and radiographically negative have no occult disease, a neck dissection is performed. Further, the patient is aware at the time of primary treatment planning, that a neck dissection will be done regardless of the response.

Dr. Peters: If there was complete resolution of the neck disease on physical examination and CT, I would not do a neck dissection. I would follow the patient closely, repeating physical examinations every 4 to 6 weeks for the first year and obtain another contrast CT scan in 6 months.

Dr. Bradford: For nodal disease greater than 3 $\mathrm{cm}$ in greatest dimension, I favor a planned modified or radical neck dissection 6 weeks after radiation therapy. I cannot comment on what type of neck dissection to perform following chemoradiation since no prospective randomized trial has proven this treatment modality to be as effective as surgery and postoperative radiation therapy for advanced stage tonsillar or oropharyngeal squamous cell carcinoma. However, for advanced laryngeal and hypopharyngeal carcinomas treated according to published protocols, ${ }^{4,5}$ planned neck dissections are advisable for residual palpable nodal disease following induction chemotherapy. ${ }^{6}$

6. If definitive radiation or chemoradiation resulted in a complete locoregional response, then at 6 months a primary site recurrence occurred that was limited to the original primary tumor site, what salvage surgical procedure would you recommend?

Dr. Peters: If a recurrence developed at the site of the original primary and he was still without evidence of recurrence in the neck, then I would resect the tonsil recurrence using a median mandibulotomy and also perform an ipsilateral modified neck dissection. The reasons for the neck dissection include the need to sample the involved nodes to rule out neck recurrence, "violation" of the neck in resecting the recurrence in the oropharynx, and the need to provide recipient vessels for the radial forearm flap that I would use for reconstruction. 
Dr. Bradford: If definitive radiation or chemoradiation resulted in a complete locoregional response and 6 months later a primary site recurrence occurred, I would perform the originally planned surgical resection and reconstruction (see \#4 above). Clearly, in this instance free tissue transfer is recommended due to the poor wound healing expected 6 months after radiation and/or chemoradiation.

Dr. Futran: At this juncture, surgical extirpation is the only potential curative modality left. Therefore, the patient would require a resection of the original tumor volume with appropriate margins by a lip splitting approach, which would include the angle of the mandible, tonsillar fossa, and necessary contiguous structures with frozen section margin control and a modified neck dissection if it hadn't been done previously on the ipsilateral side. The defect would be reconstructed with an osteofasciocutaneous sensate radial forearm free flap. The bone requirement is small and it is in a nontooth bearing area. The soft tissue attributes of the radial forearm flap make it an ideal choice for this defect. Since this patient is a high risk for further recurrence, once the wounds have sufficiently healed, consideration would be made for the use of weekly methotrexate therapy for 4-6 months.

\section{COMMENT}

Definitive radiation therapy with surgical salvage or surgery and postoperative radiation therapy remain the mainstays of treatment for tonsillar squamous cell carcinoma. All of the consultants agree that chemoradiation treatment regimens should be performed in a protocol setting. There are some tumor characteristics that may be helpful in selecting treatment, assuming the patient does not have any treatment biases. Factors that are felt to predict radiation responsiveness are tumors limited to the tonsillar fossa, superficial or exophytic tumors, and poorly differentiated or lymphoepithelioma histology. Anterior pillar lesions with retromolar trigone extension, significant tongue base extension, or trismus are preferably treated surgically (Dr. Futran). Dr. Peters bases his decision making primarily on clinical stage, and does not feel that a reliable biologic or molecular marker has been identified to help with treatment planning.

The authors vary considerably in their treatment approach for this particular patient. Dr. Peters favors chemotherapy and radiation therapy on a treatment protocol, and if this were not an option, then accelerated fraction radiation therapy. He emphasizes the necessity for close monitoring for an objective response after two cycles of chemotherapy or 50 Gy of radiation with immediate salvage for inadequate responses. His rationale for this approach is that the best functional outcome is achieved without compromising oncologic control. Dr. Bradford favors surgical resection followed by radiation therapy and possibly adjuvant chemotherapy based on histopathologic parameters. She feels that combined modality treatment to both the primary site and necks provides superior locoregional control compared with definitive radiation therapy, provides objective histopathologic criteria for entry into adjuvant therapy protocols, and that sophisticated reconstruction of the defects have reduced the surgical morbidity. Dr. Futran favors external beam radiation therapy with standard fractionation, along with a planned modified radical neck dissection. He feels that radiation therapy will provide a high likelihood of local control for this patient, and that the functional outcome will be better than primary surgery.

Another alternative not discussed is treatment with concomitant chemoradiation. A recently published randomized trial comparing radiation versus concomitant chemoradiation ${ }^{7}$ demonstrated a significant improvement in local control rates and disease-specific survival in a series of 100 patients with squamous cell cancer of the head and neck. However, subsite analysis revealed these differences to be significant for the larynx and hypopharyngeal sites, with no difference in local control or disease survival for patients with orophyaryngeal cancer.

If surgery were the given option, all the consultants would approach this tumor through a lip splitting incision and median or parmedian mandibulotomy and preserve the mandible unless involved with tumor. This provides the best exposure, and preservation of mental nerve and possibly lingual nerve function. None of the authors would perform a selective neck dissection sparing level V for a solitary 4-cm nodal metastasis. The sensate radial forearm free flap is the reconstructive option of choice.

The decision to perform a neck dissection in a neck that was originally clinically $\mathrm{N}+$ that after radiation or chemoradiation is N0 is important, since several studies have shown that there is a $25 \%-30 \%$ incidence of residual disease in the neck, even in N0, and failure rates for late sur- 
gical salvage of recurrent regional disease in this situation are significant. Dr. Futran advocates planned, post-therapy neck dissection in all patients with $\mathrm{N}+$ disease at the time of primary treatment planning. Dr. Peters is willing to closely follow a patient if there is complete resolution of the neck disease both clinically and radiographically, while Dr. Bradford recommends a planned neck dissection for all patients with nodal disease originally $3 \mathrm{~cm}$ or greater. The role of a selective neck dissection in the posttreatment neck that was originally $\mathrm{N}+$ and is now NO remains to be defined.

In the event of a local recurrence 6 months after treatment, all of the authors are in agreement with a lip splitting approach, neck dissection, and free flap reconstruction. Dr. Futran would include segmental bony resection, while Drs. Bradford and Peters would spare the mandible if uninvolved through a mandibulotomy approach. Drs. Bradford and Futran would resect at least the volume of the original tumor with margins.

The management of tonsillar carcinoma remains challenging and without a uniform consensus. Many other factors integrate into the decision making process, such as patient preferences and biases, surgeon and institutional philosophies, geographical distances and social restrictions involved with multiple visits to a tertiary care center, particularly in patients from a rural setting. In addition, definitive prospective studies comparing oncologic outcomes, costs, functional outcomes, and quality of life are needed.

\section{REFERENCES}

1. O'Brien CJ, Castle GK, Steven GN, et al. Limitations of radiotherapy in the definitive treatment of squamous carcinoma of the tonsillar fossa. Aus NZ J Surg 1992;62: 709-713.

2. Hicks WL, Kuriakose A, Loree TR, et al. Surgery versus radiation therapy as a single-modality treatment of tonsillar fossa carcinoma: the Roswell Park Cancer Institute experience. Laryngoscope 1998;108:1014-1019.

3. Esclamado RM, Burkey BB, Carroll WR, et al. The platysma myocutaneous flap: indications and caveats. Arch Otolaryngol Head Neck Surg 1994;120:32-35.

4. The Department of Veterans Affairs Laryngeal Cancer Study Group. Induction chemotherapy plus radiation compared with surgery plus radiation in patients with advanced laryngeal cancer. New Engl J Med 1991;324: $1685-1690$.

5. Lefebvre J-L, Chevalier D, Luboinski B, et al. Larynx preservation in pyriform sinus cancer: preliminary results of a European Organization for Research and Treatment of Cancer Phase III trial. J Natl Cancer Inst 1996;88: 890-899.

6. Wolf GT, Fisher SG. Effectiveness of salvage neck dissection for advanced regional metastases when induction chemotherapy and radiation are used for organ preservation. Laryngoscope 1992;102:934-939.

7. Adelstein DJ, Lavertu P, Saxton JP, et al. Mature results of a phase III randomized trial comparing concurrent chemoradiotherapy with radiation therapy alone in Stage III and IV squamous cell head and neck cancer. J Clin Oncol [Submitted]. 